\title{
Disease Detection in Birds and Animals Using Image Processing
}

\author{
Venkatesh $\mathrm{R}^{\mathrm{a}, 1}$, Rajakumar $\mathrm{S}^{\mathrm{b}}$ \\ ${ }^{\mathrm{a}} \mathrm{PG}$ Scholar, ${ }^{b}$ Professor \\ ${ }^{a, b}$ Dept. of ECE, Panimalar Engineering College, India
}

\begin{abstract}
In this research, a method is proposed and implemented for detecting and classifying the infected chicken. Dominant factors have been analyzed the health of the chicken and production of egg and many other conditions which will be beneficial in the living environment. For each processing stage, different algorithms have been implemented to estimate the infection produced in the chicken. The proposed algorithms are exploited to perform in a faster manner. The experimental results have been shown using MATLAB.
\end{abstract}

Keywords. Clustering, Chicken, Fowl pox, Poultry

\section{Introduction}

In an agricultural country like India, there is a large production of chicken. In this method, different methods have been analyzed to detect fowl pox. Digital images are used for analyzing. Anisotropic filter is used for denoising the image. MEMA is used for segmenting the image. Neural Network is used for identifying the image. To detect chicken from infection and diseases at an early stage. The detection and classification method is proposed.

\section{Related Works}

[1] Broiler chicken digestive dropping disease examination are done by using a most precise method using convolutional neutral network to identify the infection caused in poultry farms by the technique proposed. The obtained data's are compared with faster R-CNN and YOLO-V3 were analyzed.

[2] To develop a safety inspection and provide an effective nondestructive quality a hyperspectral imaging is proposed. The feature extraction is done by using machine vision imaging. It provides $90 \%$ accuracy than the existing methods.

[3]The multispectral imaging methods has been proposed to identify the inflammatory process in chicken. For feature extraction ROI characteristics has been analyzed. The threshold value is set to separate the septicemia chicken separately.[4] Hemalatha et. al. proposed a method to analyze the udder health status using Infrared Thermography. The output depends on the collected milk sample to somatic count and skin temperature of an udder surface. This methodology produces better results in specificity than the existing methods. [4] Hemalatha et.al. proposes a real time disease detection using two different approaches. They are machine using support vector and extreme learning machine. Feature extraction is done using grey level co-occurrence

\footnotetext{
${ }^{1}$ Venkatesh R, PG Scholar, Dept. of ECE, Panimalar Engineering College, India. 
matrix. The accuracy is obtained by ECM classifier. [5] Uddin et. al. proposed a detection method using Biosensors which provides a high sensitivity and doesn't require much diagnosis but it also provides only limited detection for a microorganism. [6] Liu et. al. proposed a detection method using wearable sensors by detecting the infection using real time. It can also detect different types of infected diseases. It has issues based on the weight of the sensor and life of the battery. [7] The detection and classification is taken place by using vocalization method by injecting clostridium perfringens to chickens. Neural network pattern is used for classifying the images by increasing the number of days it produces $100 \%$ accuracy.

\section{Proposed Method}

The infected chicken input image is filtered out from the impulse noise. By filtering the image, the degraded estimation function is proposed to find the noise pixels. The Anisotropic filtered image is proposed to eliminate the noise from the infected input image

The noise pixels is given by,

$$
y(i, j)=x(i, j)+n(i, j)
$$

For image enhancement the adaptive median filter [8][9] is proposed to apply in the colour and gray spaces of the image. Its main aim is to produce the quality in the images. By using histogram mean the pixel intensity values are displayed. Low pixels values are obtained from dark images and high pixel values are obtained from Bright Images[8-9]

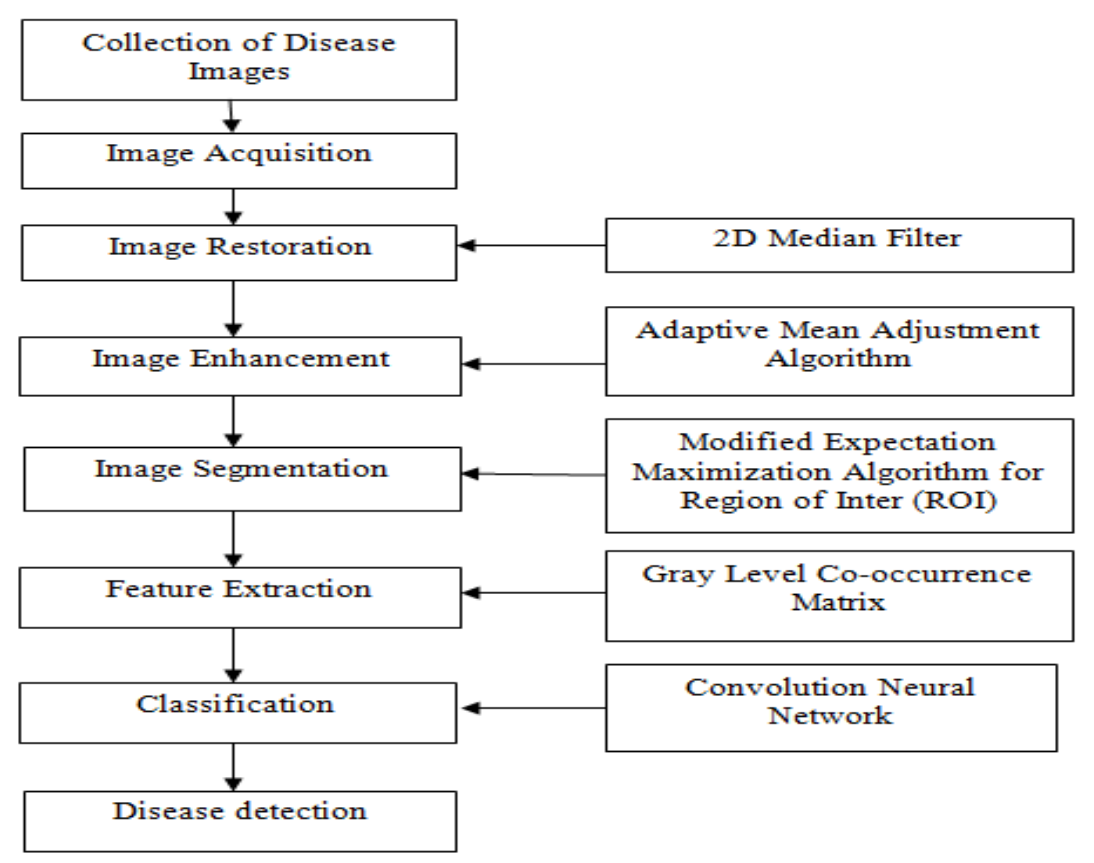

Figure 1. Block Diagram for Fowl pox 
The mean adjustment is given by

$$
x(i, j)-\frac{x_{\min }(i, j)}{x_{\max }(i, j)-x_{\min }(i, j)}
$$

For Image segmentation a modified expectation maximization algorithm is proposed. For segmentation Fuzzy C hybrid version is exploited.The main aim is to segment the image to show the infected area precisely and the image is then clustered. By setting the threshold the gray scale is converted into binary image. Grouping the infected parts are done by clustering. The feature extraction methodology contains the relevant information about the abnormality of the poultry data's available. Gray level cooccurrence matrix is proposed to extract the data. It extracts the correlation, energy and abnormality of different poultry chicken species.

The feature extraction equation for correlation is given by,

$$
F_{1}=\sum_{i=0}^{N_{g}-1} \sum_{j=0}^{N_{g}-1} \frac{\left(p_{j}\right) p(i, j)-\mu_{x} \mu_{y}}{\sigma_{x} \sigma_{y}}
$$

The feature extraction equation for energy is given by

$$
F_{2}=\sum_{i=0}^{N_{g}-1} \sum_{j=0}^{N_{g}-1}\{p(i, j)\}^{2}
$$

The feature extraction equation for homogeneity is given by

$$
F_{3}=\sum_{i=0}^{N_{g}-1} \sum_{j=0}^{N_{g}-1} \frac{p(i, j)}{1+(i-j)^{2}}
$$

\section{Results and Discussion}

Figure 2(a) shows the input image of the infection. Fig 2(b) shows a basic step of processing the image. Denoising is done to provide the soft edges and the further processing is done by clear images. For clustering the enhance and color is exploited to provide the better performance. Clustering fig 3(c) shows the infection part of the image.

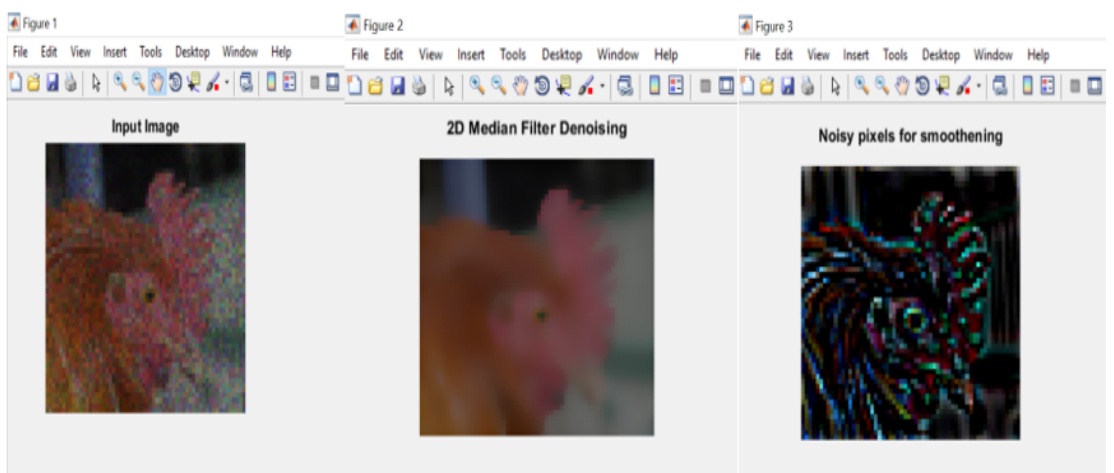

Figure 2. (a) Input Image (b) Denoising Image (c) Noise pixels from smoothening 


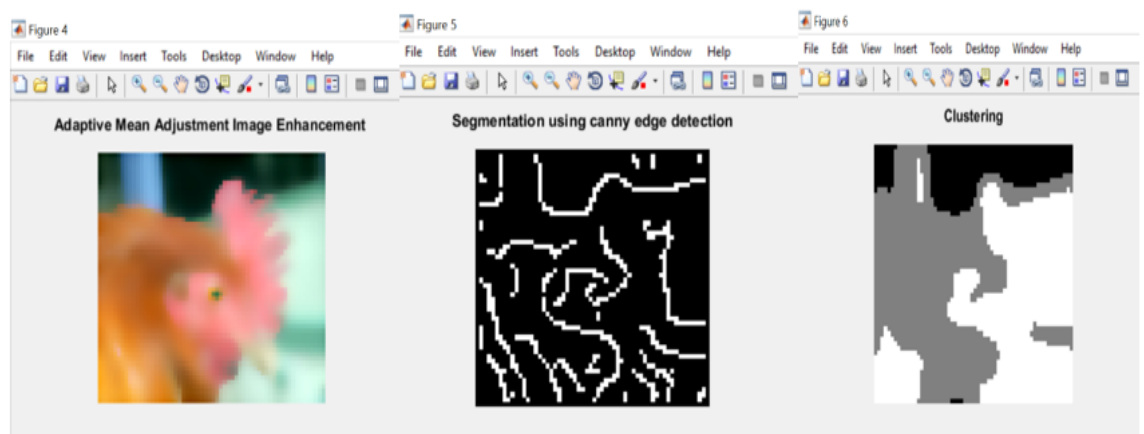

Figure 3. (a) Adaptive mean Adjustment image enhancement (b)Segmentation (c) Clustering

\section{Conclusion}

Fowl pox is a growing concern in poultry farms which appears in the external part of the body. In this paper the detection of chicken infection is proposed. The algorithms are proposed to perform in a faster manner. The performance is evaluated using clustering methods using convolutional neural network and MEM algorithm is used for Segmentation. The algorithm provides better performance than the existing methods.

\section{References}

[1] Jintao Wang, Mingxia Shen, Longshen Liu, Yi Xu, and Cedric Okinda. Recognition and Classification of Broiler Droppings Based on Deep Convolutional Neural Network. Journal of Sensors. Volume 2019 pg.10.

[2] Xiong Z, Xie A, Sun DW, Zeng XA, Liu D.Applications of hyperspectral imaging in chicken meat safety and quality detection and evaluation: a review. NCBI journal. Volume 55. Issue 9. 2015.

[3] Chun Chieh Yang, Kuanglin Chao, Yud Ren Chen. Development of multispectral Image Processing algorithms for identification of wholesome, septicemic and inflammatory Process chickens. Journal of food engineering. Volume 69 pp. 225-234. 2005

[4] Hemalatha, S Muruganand R Maheswaran.Recognition of Poultry Disease in Real Time using Extreme Learning Machine. Proceedings of International conference on Inter Displinary Research in Engineering \& Technology 2014. Pp.44-50

[5] Muhammad Ammad Uddin, Muhammad Ayaz, El Hadi Aggoune. Wireless Sensor Network: A Complete Solution for Poultry Farming. IEEE $2^{\text {nd }}$ International Symposium on Telecommunication Technologies 2014 pp.321-325.

[6] Longshen Liu, Bo Li, Ruqian Zhao, Wen Yao, Mingxia Shen, Ji Yang. A Novel Method for Broiler Abnormal Sound Detection Using WMFCC and HMM. Journal of Sensors. Volume 2020. Pp.1-7. 2020.

[7] Zaninelli M, Redaelli V, Luzi F, Bronzo V, Mitchell M, Dell'Orto V, Bontempo V, Cattaneo D, Savoini G.First Evaluation of Infrared Thermography as a Tool for the Monitoring of Udder Health Status in Farms of Dairy Cows. NCBI journal. Volume 13. Issue 92014.

[8] V.D.Ambeth Kumar and Dr.M.Ramakrishan (2012).Enhancement in Footprint Image using Diverse Filtering Technique" in the month of March for the Procedia Engineering journal (Elsevier) Journal Volume 8, No.12, 1072-1080, March 2012

[9] V.D.Ambeth Kumar, Dr.M.Ramakrishnan, V.D.Ashok Kumar and Dr.S.Malathi (2015) .Performance Improvement using an Automation System for Recognition of Multiple Parametric Features based on Human Footprint. for the International Journal of kuwait journal of science \& engineering, Vol 42, No 1 (2015), pp:109-132. 